\title{
Morita-like equivalence for fair semigroups
}

\author{
Yang Li (D), Hongxing Liu*(D) \\ School of Mathematics and Statistics, Shandong Normal University, 250014, Jinan, P.R. China
}

\begin{abstract}
In this paper, we mainly investigate Morita-like equivalence and Morita context for right fair semigroups. If two right fair semigroups $S$ and $T$ are Morita-like equivalent, that is, there is a category equivalence $F: U S-$ Act $\rightleftharpoons$ : $U T$-Act : $G$, we characterize the two functors $F$ and $G$ using Hom functor and tense product functor. Also, we investigate Morita context for right fair semigroups and obtain equivalence between two right unitary act categories.
\end{abstract}

Mathematics Subject Classification (2010). 20M50

Keywords. fair semigroup, Morita equivalence, Morita context, equivalence, functor

\section{Introduction}

Morita equivalence theory characterizes the relationship of module categories of two rings with 1. Morita theory is very useful in studying ring theory. Later, many authors tried to extend Morita equivalence theory to rings without 1. Fuller [4] obtained equivalence between a completely additive subcategory of the module category over a ring (not necessarily with 1) and a full unitary module category over a ring with 1. Abrams [1], Ánh and Márki [2] obtained Morita equivalences by replacing the usual module categories with the categories of unital modules. Xu, Shum and Turner-Smith [17] further extended Morita theory and introduced Morita-like equivalence of module categories. García, Marín and Ouyang etc. $[5,14,15]$ studied xst-rings, which is named after $\mathrm{Xu}$, Shum and TurnerSmith.

Morita theory has also been extended to semigroup theory $[3,7-13,16]$. Banaschewski [3] and Knauer [8] independently studied Morita theory of monoids and got the same conclusions. Since then, many results on Morita theory of semigroups have been obtained. Talwar [16] generalized this theory to semigroups with local units. Lawson [12] reformulated Morita theory of semigroups with local units in [16] and characterized equivalence of two semigroups with local units by joint enlargement. Recently, Liu [13] got equivalence between two subcategories of unital act categories over two arbitrary semigroups based on Morita context. Laan and Márki [11] introduced the notion of fair semigroups, which corresponds to xst-rings. They investigated Morita equivalence of these semigroups. It is worth to further consider Morita-like equivalence for fair semigroups. In this paper, we will use the tools in [15] to study Morita-like equivalence and Morita context for these semigroups.

\footnotetext{
*Corresponding Author.

Email addresses: liyang29@stu.sdnu.edu.cn (Y. Li), lhxshanda@163.com (H. Liu)

Received: 10.11.2017; Accepted: 29.08.2018
} 
The paper is constructed as follows. In Section 2, we give some basic notions and results on fair semigroups which is used in this paper. In Section 3, the Morita-like equivalence is investigated and some properties of Morita-like equivalence are obtained. We study Morita context for right fair semigroups and obtain equivalence between two unitary act categories in Section 4.

\section{Preliminaries}

Let $S$ be a semigroup. A set $M$ is a right $S$-act if there is a map from $M \times S$ to $M$, denoted $(m, s) \rightarrow m s$, such that $m(s t)=(m s) t(\forall s, t \in S, m \in M)$. If $M$ is a right $S$-act, we write $M_{S}$. If $M=M S$, we call $M$ to be unitary. If for all $m \in M$, there exists $s \in S$ such that $m s=m$, we call $M$ to be $s$-unital.

One can similarly define left $S$-acts.

For two right $S$-acts $M$ and $N, f: M \rightarrow N$ is said to be $S$-morphism if $f$ satisfies $f(m s)=f(m) s,(\forall m \in M, s \in S)$. The set of all $S$-morphisms from $M_{S}$ to $N_{S}$ is denoted by $\operatorname{Hom}_{S}(M, N)$. The set of all $S$-morphisms from $M$ to itself is denoted by $\operatorname{End}_{S}(M)$. Let $S$-Act be the category of right $S$-acts on a semigroup $S$.

The unitary right $S$-acts together with the $S$-morphisms form a full subcategory of $S$-Act, which we shall denote by $U S$-Act.

Let $S$ and $T$ be two semigroups. An $S$-T-biact is a set $M$ which is both left $S$-act and right $T$-act and $(s m) t=s(m t)$ for all $s \in S, t \in T$ and all $m \in M$. A biact is said to be unital if it is left and right unital. If $M$ and $N$ are $S$-T-biact, a map $f: M \rightarrow N$ is called a biact morphism if $f$ satisfies $f(s m)=s f(m)$ and $f(m t)=f(m) t$ for all $m \in M, s \in S, t \in T$.

An equivalence $\rho$ on $M_{S}$ is a congruence if for all $s \in S, m, n \in M$,

$$
(m, n) \in \rho \Rightarrow(m s, n s) \in \rho .
$$

If $\rho$ is a congruence on $M$, then $M / \rho$ is also a right $S$-act. The act $M / \rho$ is called a quotient act.

For a right $S$-act $A_{S}$ and a left $S$-act ${ }_{S} B$, the tensor product $A \otimes_{S} B$ exists. In fact, $A \otimes_{S} B=(A \times B) / \sigma$, where $\sigma$ is the congruence on $A \times B$ generated by

$$
\mathcal{R}=\{((x s, y),(x, s y)) \mid x \in A, y \in B, s \in S\} .
$$

We denote the element $(x, y) \sigma$ of $A \otimes_{S} B$ by $x \otimes y$.

By Proposition 1.4.10 of [6], we have that $a \otimes b=c \otimes d \Longleftrightarrow(a, b)=(c, d)$ or there is a sequence

$$
(a, b)=\left(x_{1}, y_{1}\right) \rightarrow\left(x_{2}, y_{2}\right) \rightarrow \cdots \rightarrow\left(x_{n}, y_{n}\right)=(c, d)
$$

such that either $\left(\left(x_{i}, y_{i}\right),\left(x_{i+1}, y_{i+1}\right)\right) \in T$ or $\left(\left(x_{i+1}, y_{i+1}\right),\left(x_{i}, y_{i}\right)\right) \in T$, where $1 \leq i \leq$ $n-1$.

If $A$ is a right $S$-act and $B$ is an $S$-T-biact, then $A \otimes_{S} B$ is a right $T$-act with

$$
(a \otimes b) t=a \otimes b t ;
$$

similarly, if $A$ is a $T$-S-biact and $B$ is a left $S$-act, then $A \otimes_{S} B$ is a left $T$-act with

$$
t(a \otimes b)=t a \otimes b
$$

(Proposition 3.1, [16]).

Let $S, T$ be two semigroups. For an $S$-T-biact ${ }_{S} M_{T}$ and a left $S$-act ${ }_{S} N$, we have that $\operatorname{Hom}_{S}(M, N)$ is a left $T$-act with action $(t \cdot f)(m)=f(m \cdot t)$; for an $S$-T-biact ${ }_{S} M_{T}$ and a right $S$-act $N_{T}$, we have that $\operatorname{Hom}_{T}(M, N)$ is a right $S$-act with action $(f \cdot s)(m)=f(s \cdot m)$; for a right $S$-act $M_{S}$ and a $T$-S-biact ${ }_{T} N_{S}$, we have that $\operatorname{Hom}_{S}(M, N)$ is a left $T$-act with action $(t \cdot f)(m)=t \cdot f(m)$; for a left $T$-act ${ }_{T} M$ and a $T$-S-biact ${ }_{T} N_{S}$, we have that $\operatorname{Hom}_{T}(M, N)$ is a right $S$-act with action $(f \cdot s)(m)=f(m) \cdot s$. 
A functor $F$ is said to be faithful if it is injective. A semigroup $S$ is called factorisable if every element of $S$ is a product of two elements.

Corresponding to the notion of xst-rings investigated by García and Marín in [5], Laan and Márki [11] introduced the notion of fair semigroups.

Definition 2.1. [11] Let $S$ be a semigroup. If every subact of an unitary right $S$-act is unital, $S$ is said to be a right fair semigroup.

Similarly, we can define left fair semigroups. A fair semigroup is a semigroup $S$ that is both a left fair semigroup and a right fair semigroup.

By Proposition 2.3 in [11], $S$ is a fair semigroup if and only if every right unital $s$-act is $s$-unital.

An ideal $I$ of $S$ is said to be unitary if $I$ is unitary as $S$-act. Let $U\left(S_{S}\right)$ be the disjoint union of all right unitary ideals of $S$. It is obvious that $U\left(S_{S}\right)$ is the largest right unitary ideal of $S$. Similarly, we can define the left ideal $U\left({ }_{S} S\right)$. If $S$ is factorisable, then $U\left(S_{S}\right)=U\left({ }_{S} S\right)=S$.

Let $S$ be a right fair semigroup. Laan and Márki [11] proved that $U\left(S_{S}\right)$ is a two-sided ideal and $U\left({ }_{S} S\right)=U\left(S_{S}\right)$. We denote $U\left({ }_{S} S\right)=U\left(S_{S}\right)$ by $U(S)$. By Corollary 2.13 in [11], we have

$$
U(S)=\{s \in S \mid s=s u=v s \text { for some } u, v \in S\} .
$$

The set $U(S)$ is said to be the unitary part of $S$.

A right $S$-act $M_{S}$ is nonsingular, if $m=m^{\prime}\left(m, m^{\prime} \in M\right)$ whenever $m s=m^{\prime} s$ for all $s \in S$ ([11]). A semigroup $S$ has common weak right local units, if for all $s, t \in S$, there is an element $u \in S$ such that $s=s u$ and $t=t u([11])$. Let $S$ be a fair semigroup such that $U(S)$ has common weak right local units. By Proposition 3.2 in [11], every act in $U S$-Act is nonsingular. Define $\mu_{M}: M \otimes_{S} S \rightarrow M$ by $m \otimes s \mapsto m s$. Let

$$
F S \text {-Act }=\{M \in S \text {-Act } \mid M \otimes S \cong M\} .
$$

If $S$ has common weak right local units, then $F S$-Act $=U S$-Act ([11]).

We generalize the definition of Morita-like equivalence to semigroup theory.

Definition 2.2. Let $S$ and $T$ be two fair semigroups. $S$ and $T$ are Morita-like equivalent if $U S$-Act is equivalent to $U T$-Act.

\section{Morita-like equivalence for fair semigroups}

Let $M_{S}$ be a right $S$-act. Denote by $U\left(M_{S}\right)$ the union of all unitary subacts of $M_{S}$. Then $U(M)$ is the largest subact of $M_{S}$ which is unitary. The following proposition gives a characterization of $U\left(M_{S}\right)$, which is analogous to Proposition 2.2 in [15].

Proposition 3.1. Let $S$ be a fair semigroup. For $M \in U S$-Act, we have $U\left(M_{S}\right)=$ $M U\left(S_{S}\right)$. In particular, if $S$ is a factorisable right fair semigroup, then $U\left(M_{S}\right)=M S$.

Proof. Since $M U\left(S_{S}\right)$ is a unitary subact of $M_{S}$, we have that $M U\left(S_{S}\right) \subseteq U\left(M_{S}\right)$.

If $N$ is a unitary subact of $M_{S}$, we get $N U\left(S_{S}\right) \subseteq N S=N$. Assume $N U\left(S_{S}\right) \neq N$. There exitsts an element $x \in N=N S$ but $x \notin N U(S)$. Hence, we have $x=n_{1} s_{1}$, where $n_{1} \in N$ and $s_{1} \in S$. Since $U(S)$ is a right ideal and $n_{1} s_{1} \notin N U(S)$, we have $n_{1} \in N \backslash N U(S)$ and $s_{1} \notin U(S)$. For $n_{1} \in N S \backslash N U(S)$, we have $n_{1}=n_{2} s_{2}$, where $n_{2} \in N$ and $s_{2} \in S \backslash U(S)$.

Continuing in this way, we can get two sequences $n_{1}, n_{2}, \ldots, n_{k}, \ldots \in N$ and $s_{1}, s_{2}, \ldots, s_{k}, \ldots \in S$ such that $n_{k} s_{k} s_{k-1} \cdots s_{1} \notin N U\left(S_{S}\right)$, for any positive integral $k$. By Theorem 2.6 of [11], we can find some $u \in S$ such that $s_{k} \cdots s_{1}=s_{k} \cdots s_{1} u$. By Lemma 2.12 (2) in [11], we get $s_{k} s_{k-1} \cdots s_{1} \in U(S)$. This is a contradiction. Hence, $N U\left(S_{S}\right)=N$.

For any unitary subact $N$ of $M_{S}$, we have

$$
N=N U\left(S_{S}\right) \subseteq M U\left(S_{S}\right) \subseteq U\left(M_{S}\right) .
$$


Particularly, let $N=U\left(M_{S}\right)$, we have $U\left(M_{S}\right)=M U\left(S_{S}\right)$.

If $R$ is a right xst-ring and $M$ is an $R$-module, there is a natural equivalence $M \cong$ $\operatorname{Hom}_{R}(U(R), M) U(R)([15])$. The following statement gives a similar result for right fair semigroup $S$, where $U(S)$ has common weak right local units.

Proposition 3.2. Let $S$ be a right fair semigroup such that $U(S)$ has common weak right local units. For $M \in U S$-Act, define

$$
\theta_{M}: M_{S} \longrightarrow \operatorname{Hom}_{S}\left(U\left(S_{S}\right), M_{S}\right) U\left(S_{S}\right)
$$

by putting $\theta_{M}(x)(s)=x s$, where $x \in M$ and $s \in U\left(S_{S}\right)$, then $\theta_{M}$ is a natural $S$ isomorphism. Moreover,

$$
\theta: I_{U S-A c t} \longrightarrow \operatorname{Hom}_{S}\left(U\left(S_{S}\right),-\right) U\left(S_{S}\right)
$$

is a natural equivalence.

Particularly, if $S$ is a factorisable right fair semigroup, we have

$$
\theta: I_{U S-A c t} \longrightarrow \operatorname{Hom}_{S}(S,-) S
$$

is a natural equivalence.

Proof. Firstly, we show that $\theta_{M}$ is injective. For all $x, y \in M$, if $\theta_{M}(x)=\theta_{M}(y)$, we have $\theta_{M}(x)(s)=\theta_{M}(y)(s)$, for all $s \in U\left(S_{S}\right)$. That is, $x s=y s$. Hence, we get $x=y$ by Proposition 3.2 in [11].

Next, we show that $\theta_{M}$ is surjective. We know that $\operatorname{Hom}_{S}\left(U\left(S_{S}\right), M_{S}\right) U\left(S_{S}\right)$ is the largest unitary subact of $\operatorname{Hom}_{S}\left(U\left(S_{S}\right), M_{S}\right)$ by Proposition 3.1. For all

$g \in \operatorname{Hom}_{S}\left(U\left(S_{S}\right), M_{S}\right) U\left(S_{S}\right)$, suppose that $g=g^{\prime} s^{\prime}$, where $g^{\prime} \in \operatorname{Hom}_{S}\left(U(S), M_{S}\right), s^{\prime} \in$ $U\left(S_{S}\right)$, there exits $u \in S$ satisfying $s^{\prime}=s^{\prime} u$ by Lemma 2.12 in [11]. Hence,

$$
g=g^{\prime} s^{\prime}=g^{\prime} s^{\prime} u=g u .
$$

Since $S$ is a right fair semigroup, there exists a positive integer $n$ such that $u^{n} \in U\left(S_{S}\right)$ by Corollary 2.10 in [11]. Also, we have $g=g u^{n}$. Therefore,

$$
g\left(x^{\prime}\right)=\left(g u^{n}\right)\left(x^{\prime}\right)=g\left(u^{n} x^{\prime}\right)=g\left(u^{n}\right) x^{\prime}=\theta_{M}\left(g\left(u^{n}\right)\right)\left(x^{\prime}\right),
$$

where $x^{\prime} \in U\left(S_{S}\right)$. This concludes that $g=\theta_{M}\left(g\left(u^{n}\right)\right)$ and so $\operatorname{Hom}_{S}\left(U\left(S_{S}\right), M_{S}\right) U(S) \subseteq$ $\operatorname{Im} \theta_{M}$.

Since $\operatorname{Im} \theta_{M}$ is a subact of $\operatorname{Hom}_{S}\left(U\left(S_{S}\right), M_{S}\right) U\left(S_{S}\right)$, we have

$$
\operatorname{Im} \theta_{M}=\operatorname{Hom}_{S}\left(U\left(S_{S}\right), M_{S}\right) U\left(S_{S}\right) .
$$

This implies that $\theta_{M}$ is surjective.

Clearly, $\theta_{M}$ is a natural isomorphism.

Remark 3.3. (i) If $S$ is a factorisable right fair semigroup, then

$$
S_{S} \cong \operatorname{Hom}_{S}\left(S, S_{S}\right) S=\operatorname{End}_{S}\left(S_{S}\right) S .
$$

(ii) Let $S$ be a right fair semigroup. For $M \in U S$-Act, if ${ }_{T} M_{S}$ is a $T$-S-biact, then

$$
\theta_{M}: M_{S} \longrightarrow \operatorname{Hom}_{S}\left(U\left(S_{S}\right), M_{S}\right) U\left(S_{S}\right)
$$

is a $T$ - $S$-isomorphism.

Let $\left\{M_{i} \mid i \in I\right\}$ be a family of $S$-Act, the coproduct $\coprod_{i \in I} M_{i}$ is the disjoint union of $M_{i}$.

Let $S$ be a semigroup. For all $M \in U S$-Act, if $M$ is an epimorphic image of $\amalg P$, we call the unitary $S$-act $P$ to be a generator of $U S$-Act.

Proposition 3.4. Let $S$ be a right fair semigroup, and $P$ be an object in US-Act. Then the following are equivalent:

(1) $P$ is a generator of $U S$-Act.

(2) The functor $\operatorname{Hom}_{S}\left(P_{S},-\right)$ is faithful. 
Proof. (1) $\Longrightarrow(2)$ For $M, N \in U S$-Act, suppose that $f$ and $g$ are two $S$-morphisms from $M$ to $N$ such that $\operatorname{Hom}(P, f)=\operatorname{Hom}(P, g)$. For all $m \in M$, since $P$ is a generator, there is an $S$-morphism $\mu: \amalg P \longrightarrow M$ such that $m=\mu(p)$. Hence

$$
f(m)=f \mu(m)=g \mu(m)=g(m) .
$$

This implies that $f=g$. Therefore, $\operatorname{Hom}(P,-)$ is faithful.

$(2) \Longrightarrow(1)$ Let $I=\operatorname{Hom}_{S}\left(P_{S}, S_{S}\right)$ and $\mu: P^{(I)} \longrightarrow S$ be a morphism indexed by the elements of $I$. If $\mu$ is not epic, we have

$$
\operatorname{Hom}(P, S) \longrightarrow \operatorname{Hom}(P, S / \operatorname{Im} \mu)
$$

For all $f_{1}, f_{2} \in \operatorname{Hom}(P, S)$, we have $\mu f_{1}=\mu f_{2}$. This is contrary to that $\operatorname{Hom}(P,-)$ is faithful.

By Proposition 3.5 in [11], we can now establish the following result:

Proposition 3.5. Let $S$ be a right fair semigroup such that $S^{\prime}=U\left(S_{S}\right)$ has common weak right local units.

(1) Every right unitary $S$-act is a right unitary $S^{\prime}$-act, and vice versa.

(2) $\operatorname{Hom}_{S}(M, N)=\operatorname{Hom}_{S^{\prime}}(M, N)$, for any $M, N \in U S$-Act.

(3) $S^{\prime}$ is a generator in $U S$-Act.

Proof. We can obtain (1) and (2) by the proof of Proposition 3.5 in [11].

(3) For all $M \in U S$-Act, we have that $M$ is also a unitary right $S^{\prime}$-act by (1). So $M$ is $s$-unital by Proposition 2.3 in [11]. Let $g: S^{\prime M} \longrightarrow M$ defined by $s \mapsto m s$. This proves that $S^{\prime}$ generates $M$.

Let $S$ and $T$ be two semigroups. If ${ }_{S} P_{T}$ and ${ }_{T} Q_{S}$ are biacts, $\tau: P \otimes_{T} Q \longrightarrow S$ and $\mu: Q \otimes_{S} P \longrightarrow T$ are biact morphisms correspondingly as written

$$
\tau(p \otimes q)=<p, q>, \quad \mu(q \otimes p)=[q, p]
$$

such that

$$
<p_{1}, q>\cdot p_{2}=p_{1} \cdot\left[q, p_{2}\right], \quad\left[q_{1}, p\right] \cdot q_{2}=q_{1} \cdot<p, q_{2}>,
$$

for all $p, p_{1}, p_{2} \in P$ and $q, q_{1}, q_{2} \in Q$. The set $\left(S, T,{ }_{S} P_{T},{ }_{T} Q_{S}, \tau, \mu\right)$ is said to be a Morita context.

Note that $U(T)$ is a unitary $U(T)$-act, it follows that $U(T)$ is also $s$-unital as $U(T)$ act. That is, for all $t \in U(T)$, there is $t^{\prime} \in U(T)$ such that $t=t t^{\prime}$. Hence, for all $f \in \operatorname{Hom}_{T}(Q, M) U(T)$, there is $t \in U(T)$ such that $f t=f$.

Corresponding to Theorem 3.1 in [15], we give the following theorem. We use a different way to prove this result.

Theorem 3.6. Let $S$ and $T$ be two semigroups. Suppose $\left(S, T,{ }_{S} P_{T},{ }_{T} Q_{S}, \tau, \mu\right)$ is a Morita context, where $\tau: P \otimes_{T} Q \longrightarrow S$ and $\mu: Q \otimes_{S} P \longrightarrow T$ are biact morphisms such that $\operatorname{Im} \tau=U\left(S_{S}\right)$ and $\operatorname{Im} \mu=U\left(T_{T}\right)$. Then we have the following natural functor isomorphisms:

$$
-\otimes_{S} P \cong H_{S}\left(Q_{S},-\right) U\left(T_{T}\right), \quad-\otimes_{T} Q \cong \operatorname{Hom}_{T}\left(P_{T},-\right) U\left(S_{S}\right) .
$$

Proof. Define a map $\tau_{M}: M \otimes_{S} P \longrightarrow \operatorname{Hom}_{S}\left(Q_{S}, M_{S}\right) U\left(T_{T}\right)$ by $m \otimes p \longmapsto m \tau(p \otimes-) t$, that is

$$
\tau_{M}(m \otimes p)=m \tau(p \otimes-) t,
$$

where $t \in U(T)$ such that $m \tau(p \otimes-) t=m \tau(p \otimes-)$.

We claim that $\tau_{M}$ is well-defined.

Suppose that $m \otimes p=m^{\prime} \otimes p^{\prime}$, we have $(m, p)=\left(m^{\prime}, p^{\prime}\right)$ in which case

$$
m \tau(p \otimes-) t=m^{\prime} \tau\left(p^{\prime} \otimes-\right) t
$$


or

$$
\begin{array}{cc}
m=m_{1} s_{1}, & s_{1} p=t_{1} p_{1}, \\
m_{1} t_{1}=m_{2} s_{2}, & s_{2} p_{1}=t_{2} p_{2}, \\
\cdots & \cdots \\
m_{n-1} t_{n-1}=m_{n} s_{n}, & s_{n} p_{n-1}=t_{n} p^{\prime}, \\
m_{n} t_{n}=m^{\prime}, &
\end{array}
$$

where $m_{1}, m_{2}, \cdots, m_{n} \in M, p_{1}, p_{2}, \cdots, p_{n} \in P, s_{1}, s_{2}, \cdots, s_{n}, t_{1}, t_{2}, \cdots, t_{n} \in S$. Then

$$
\begin{aligned}
m \tau(p \otimes-) & =m_{1} s_{1} \tau(p \otimes-)=m_{1} \tau\left(s_{1} p_{1} \otimes-\right)=m_{1} \tau\left(t_{1} p_{1} \otimes-\right) \\
& =m_{1} t_{1} \tau\left(p_{1} \otimes-\right)=m_{2} s_{2} \tau\left(p_{1} \otimes-\right) \\
& =\cdots \cdots \\
& =m_{n} s_{n} \tau\left(p_{n-1} \otimes-\right)=m_{n} \tau\left(s_{n} p_{n-1} \otimes-\right) \\
& =m_{n} \tau\left(t_{n} p^{\prime} \otimes-\right)=m_{n} t_{n} \tau\left(p^{\prime} \otimes-\right)=m^{\prime} \tau\left(p^{\prime} \otimes-\right) .
\end{aligned}
$$

Define $\bar{\mu}: \operatorname{Hom}_{S}\left(Q_{S}, M_{S}\right) U\left(T_{T}\right) \longrightarrow M \otimes_{S} P$ by $f \longmapsto f(q) \otimes p$, where $f \in \operatorname{Hom}_{S}\left(Q_{S}, M_{S}\right)$, $t \in U(T)$ such that $f t=f$ and $t=\mu(q \otimes p)$. Suppose $f t_{1}=f=f t_{2}$, where $t_{1}=$ $\mu(q \otimes p), t_{2}=\mu\left(q^{\prime} \otimes p^{\prime}\right)$. We have

$$
\begin{aligned}
f(q) \otimes p & =(f \cdot t)(q) \otimes p=f(t \cdot q) \otimes p=f\left(\mu\left(q^{\prime} \otimes p^{\prime}\right) q\right) \otimes p \\
& =f\left(q^{\prime} \tau\left(p^{\prime} \otimes q\right)\right) \otimes p=f\left(q^{\prime}\right) \tau\left(p^{\prime} \otimes q\right) \otimes p \\
& =f\left(q^{\prime}\right) \otimes p^{\prime} \mu(q \otimes p)=f\left(q^{\prime}\right) \otimes p^{\prime} \mu\left(q^{\prime} \otimes p^{\prime}\right) \\
& =f\left(q^{\prime}\right) \tau\left(p^{\prime} \otimes q^{\prime}\right) \otimes p^{\prime}=f\left(q^{\prime} \tau\left(p^{\prime} \otimes q^{\prime}\right)\right) \otimes p^{\prime} \\
& =f\left(t q^{\prime}\right) \otimes p^{\prime}=f t\left(q^{\prime}\right) \otimes p^{\prime}=f\left(q^{\prime}\right) \otimes p^{\prime} .
\end{aligned}
$$

So $\bar{\mu}$ is well-defined.

For all $m \otimes p \in M \otimes_{S} P$, we have

$$
\begin{aligned}
\overline{\mu \tau}(m \otimes p) & =\bar{\mu}(m \tau(p \otimes-) t)=\bar{\mu}\left(m \tau(p \otimes-) \mu\left(q^{\prime} \otimes p^{\prime}\right)\right) \\
& =m \tau\left(p \otimes q^{\prime}\right) \otimes p^{\prime}=m \otimes \tau\left(p \otimes q^{\prime}\right) p^{\prime} \\
& =m \otimes p \mu\left(q^{\prime} \otimes p^{\prime}\right)=(m \otimes p) t,
\end{aligned}
$$

where $t=\mu\left(q^{\prime} \otimes p^{\prime}\right)$ with $m \tau(p \otimes-) t=m \tau(p \otimes-)$. Hence, we have $\overline{\mu \tau}=I_{m \otimes p}$.

For all $f \in \operatorname{Hom}_{T}(Q, M) U(T)$, suppose $f t=f$ and $t=\mu(q \otimes p)$. We have

$$
\overline{\tau \mu}(f)=\overline{\tau \mu}(f t)=\bar{\tau}(f(q) \otimes p)=f(q) \tau(p \otimes-) t .
$$

For all $x \in Q$, we have

$$
f(q) \tau(p \otimes x)=f(q \tau(p \otimes x))=f(\mu(q \otimes p) x)=f(t x)=f t(x) .
$$

Thus, $f(q) \tau(p \otimes-)=f t(-)=f(-)$. It concludes $\overline{\tau \mu}=I_{\operatorname{Hom}_{S}\left(Q_{S}, M_{S}\right) U\left(T_{T}\right)}$.

The naturality is obvious. Hence, we get $-\otimes_{S} P \cong \operatorname{Hom}_{S}\left(Q_{S},-\right) U\left(T_{T}\right)$.

Similarly, we have $-\otimes_{T} Q \cong \operatorname{Hom}_{T}\left(P_{T},-\right) U\left(S_{S}\right)$.

We can obtain the following corollary by the above theorem.

Corollary 3.7. Let $S$ and $T$ be two fair semigroups. Assume $\left(S, T,{ }_{S} P_{T},{ }_{T} Q_{S}, \tau, \mu\right)$ is a Morita context, where ${ }_{S} P_{T}$ and ${ }_{T} Q_{S}$ are unitary biacts, $\mu: Q \otimes_{S} P \rightarrow T$ and $\tau: P \otimes_{T} Q \rightarrow$ $S$ are biact morphisms such that $\operatorname{Im} \tau=U\left(S_{S}\right)$ and $\operatorname{Im} \mu=U\left(T_{T}\right)$. We have the following natural functor isomorphisms:

$$
\begin{aligned}
& -\otimes_{S} P \cong \operatorname{Hom}_{S}\left(Q_{S},-\right) U(T), \quad-\otimes_{T} Q \cong \operatorname{Hom}_{T}\left(P_{T},-\right) U(S), \\
& Q \otimes_{S}-\cong U(T) \operatorname{Hom}_{S}\left({ }_{S} P,-\right), \quad P \otimes_{T}-\cong U(S) \operatorname{Hom}_{T}(T Q,-) .
\end{aligned}
$$


Theorem 3.3 in [15] gives some properties of Morita-like equivalence of right-xst rings. We shall give the corresponding properties for right fair semigroups.

Theorem 3.8. Let $S$ and $T$ be two right fair semigroups such that $U\left(S_{S}\right)$ and $U\left(T_{T}\right)$ have common weak right local units. Assume $S$ and $T$ are Morita-like equivalence via $F: U S$-Act $\rightleftharpoons U T$-Act $: G$. Let $P=F\left(U\left(S_{S}\right)\right)$ and $Q=G\left(U\left(T_{T}\right)\right)$. Then

(1) $P_{T}$ and $Q_{S}$ are respectively generators in $U T$-Act and $U S$-Act.

(2) $\operatorname{End}\left(P_{T}\right) U\left(S_{S}\right) \cong U\left(S_{S}\right)$ and $\operatorname{End}\left(Q_{S}\right) U\left(T_{T}\right) \cong U\left(T_{T}\right)$ as semigroups.

(3) ${ }_{S} P_{T}$ and ${ }_{T} Q_{S}$ are unitary biacts.

(4) ${ }_{S} P_{T} \cong \operatorname{Hom}_{S}\left(Q_{S}, U\left(S_{S}\right)\right) U\left(T_{T}\right)$ and ${ }_{T} Q_{S} \cong \operatorname{Hom}_{T}\left(P_{T}, U\left(T_{T}\right)\right) U\left(S_{S}\right)$.

(5) $F \approx \operatorname{Hom}_{S}\left(Q_{S},-\right) U\left(T_{T}\right)$ and $G \approx \operatorname{Hom}_{T}\left(P_{T},-\right) U\left(S_{S}\right)$.

(6) ${ }_{S} P_{T}$ and ${ }_{T} Q_{S}$ induce a Morita context $\left(S, T,{ }_{S} P_{T},{ }_{T} Q_{S}, \tau, \mu\right)$ such that $\tau$ and $\mu$ are biact homorphisms such that $\operatorname{Im} \tau=U\left(S_{S}\right)$ and $\operatorname{Im} \mu=U\left(T_{T}\right)$, respectively.

Moreover, if we define multiplications in $Q \otimes_{S} P$ and $P \otimes_{T} Q$, respectively:

$(y \otimes x)\left(y^{\prime} \otimes x^{\prime}\right)=y \otimes \tau\left(x \otimes y^{\prime}\right) x^{\prime},(x \otimes y)\left(x^{\prime} \otimes y^{\prime}\right)=x \otimes \mu\left(y \otimes x^{\prime}\right) y^{\prime}$,

where $x, x^{\prime} \in P$ and $y, y^{\prime} \in Q$, then $Q \otimes_{S} P$ and $P \otimes_{T} Q$ are semigroups, and $\mu$ and $\tau$ are semigroup homomorphisms.

(7) $F \approx-\otimes_{S} P$ and $G \approx-\otimes_{T} Q$.

Proof. We denote $U\left(S_{S}\right)=S^{\prime}$ and $U\left(T_{T}\right)=T^{\prime}$.

(1) For $M, N \in U T$-Act. Suppose $f, g \in \operatorname{Hom}_{T}(M, N)$ and $f \neq g$. Since

$$
G: \operatorname{Hom}_{T}(M, N) \longmapsto \operatorname{Hom}_{S}(G(M), G(N))
$$

is an abelian group isomorphism, we have $G(f) \neq G(g)$. By Proposition 3.5, we have that $S^{\prime}$ is a generator in $U S$-Act. It follows that $\operatorname{Hom}_{S}\left(S_{S}^{\prime},-\right)$ is faithful by Proposition 3.4. Hence, $\operatorname{Hom}_{S}\left(S^{\prime},-\right) G(f) \neq \operatorname{Hom}_{S}\left(S^{\prime},-\right) G(g)$. Therefore, $F(G(f)) F(h) \neq F(G(g)) F(h)$, where $h$ is a morphism from $S^{\prime}$ to $G(M)$. That is, $f F(h) \neq g F(h)$. This concludes that $\operatorname{Hom}\left(F\left(S^{\prime}\right),-\right)$ is faithful and $P=F\left(S^{\prime}\right)$ is a generator.

Similarly, $Q_{S}$ is a generator in $U S$-Act.

(2) Since $F: U S$-Act $\rightleftharpoons U T$-Act $: G$ is category equivalence and $Q=G\left(T^{\prime}\right)$, we have $\operatorname{End}_{T}\left(T^{\prime}\right) \cong \operatorname{End}_{T}(Q)$. By Proposition 3.2, we obtain $\operatorname{End}_{T}\left(T_{T}^{\prime}\right) T^{\prime} \cong T^{\prime}$. Therefore, $\operatorname{End}_{S}\left(Q_{S}\right) T^{\prime} \cong T^{\prime}$ as semigroups.

Similarly, $\operatorname{End}_{T}\left(P_{T}\right) S^{\prime} \cong S^{\prime}$.

(3) In virtue of (1) and (2), we know that ${ }_{S} P_{T}$ and ${ }_{T} Q_{S}$ are unitary biacts.

(4) Since

$$
\operatorname{Hom}_{S}\left(Q_{S}, S_{S}^{\prime}\right) \cong \operatorname{Hom}_{T}\left(F\left(Q_{S}\right), F\left(S_{S}^{\prime}\right)\right) \cong \operatorname{Hom}_{T}\left(T_{T}^{\prime}, P_{T}\right),
$$

we obtain

$$
\operatorname{Hom}_{S}\left(Q_{S}, S_{S}^{\prime}\right) T^{\prime} \cong \operatorname{Hom}_{T}\left(F\left(Q_{S}\right), F\left(S_{S}^{\prime}\right)\right) T^{\prime} \cong \operatorname{Hom}_{T}\left(T_{T}^{\prime}, P_{T}\right) T^{\prime} \cong{ }_{S} P_{T},
$$

in virtue of Proposition 3.2.

Similarly, we have

$$
\operatorname{Hom}_{S}\left(Q_{S}, S_{S}^{\prime}\right) T^{\prime} \cong \operatorname{Hom}_{T}\left(F\left(Q_{S}\right), F\left(S_{S}^{\prime}\right)\right) T^{\prime} \cong \operatorname{Hom}_{T}\left(T_{T}^{\prime}, P_{T}\right) T^{\prime} \cong{ }_{S} P_{T} .
$$

(5) By Proposition 3.2, for all $M \in U S$-Act, we can get the natural isomorphisms

$$
F\left(M_{S}\right) \cong \operatorname{Hom}_{T}\left(T_{T}^{\prime}, F\left(M_{S}\right)\right) T^{\prime} \cong \operatorname{Hom}_{T}\left(G\left(T_{T}^{\prime}\right), G F\left(M_{S}\right)\right) T^{\prime} \cong \operatorname{Hom}_{S}\left(Q_{S}, M_{S}\right) T^{\prime}
$$

This implies that $F \approx \operatorname{Hom}_{S}\left(Q_{S},-\right) T^{\prime}$ and $G \approx \operatorname{Hom}_{T}\left(P_{T},-\right) S^{\prime}$.

(6) By (2) and (4), we can identify ${ }_{T} Q_{S}$ with $\operatorname{Hom}_{T}\left(P_{T}, T_{T}^{\prime}\right) S^{\prime}$ and $S^{\prime}$ with $\operatorname{End}\left(P_{T}\right) S^{\prime}$. Set

$$
<,>: Q \times P \longrightarrow T^{\prime}
$$


by $\langle y, x>=y x$, and

$$
[,]: P \times Q \longrightarrow S^{\prime}
$$

by $[x, y] x^{\prime}=x<y, x^{\prime}>=x\left(y x^{\prime}\right)$ for $x, x^{\prime} \in P$ and $y \in Q$.

Clearly, $<,>$ and $[$,$] are both balanced mappings.$

Define

$$
\tau: P \otimes_{T} Q \longrightarrow S^{\prime}, \mu: Q \otimes_{S} P \longrightarrow T^{\prime}
$$

by

$$
\tau(x \otimes y)=[x, y], \mu(y \otimes x)=<y, x>=y x .
$$

Since ${ }_{S} P_{T}$ and ${ }_{T} Q_{S}$ are unitary biacts, $\tau$ and $\mu$ are biact morphisms. We can easily check that $\left(S, T,{ }_{S} P_{T},{ }_{T} Q_{S}, \tau, \mu\right)$ is a Morita context.

Since $P_{T}$ is a generator in $U T$-Act, we have

$$
T_{T}^{\prime}=\bigcup_{g \in \operatorname{Hom}_{T}\left(P_{T}, T_{T}^{\prime}\right)} g P .
$$

For all $t \in T^{\prime}$, there exist $x \in P$ and $g \in \operatorname{Hom}_{T}\left(P_{T}, T_{T}^{\prime}\right)$ such that $t=g x$. Since ${ }_{S} P$ is a unitary left $S$-act, we can write $x=s x^{\prime}$, for some $s \in S, x^{\prime} \in P$. Hence,

$$
t=g\left(s x^{\prime}\right)=<g, s x^{\prime}>=<g s, x^{\prime}>=\mu\left(g s \otimes x^{\prime}\right) .
$$

So $\mu$ is surjective.

Since $Q_{S}$ is a generator in $U S$-Act, for all $s \in S^{\prime}$, we have $s=f y$, where $f \in$ $\operatorname{Hom}_{S}\left(Q_{S}, S_{S}^{\prime}\right)$ and $y \in Q$. As $Q$ is a unitary left $T^{\prime}$-act, there exist $t \in T^{\prime}$ and $y^{\prime} \in Q$ such that $y=t y^{\prime}$. Therefore, we can write $s=f t y^{\prime}$. Since $T^{\prime}$ has common weak right local units, there exists $e \in T^{\prime}$ such that $t e=t$ for all $t \in T^{\prime}$. have

Let $e=\mu(q \otimes p)$, where $q \in Q$ and $p \in P$. Since $f t \in \operatorname{Hom}_{S}\left(Q_{S}, S_{S}\right) T^{\prime}$ and $f t q \in S$, we

$$
s=(f t e) y^{\prime}=\left(f t<q, p>y^{\prime}=f t q\left[p, y^{\prime}\right]=\left[f t q p, y^{\prime}\right]=\tau\left((f t q) p \otimes y^{\prime}\right) .\right.
$$

So $\tau$ is surjective.

In addition, we can verify that $Q \otimes_{S} P$ and $P \otimes_{T} Q$ are semigroups about the multiplications defined above. So $\mu$ and $\tau$ are semigroup isomorphisms.

(6) From the proof process of Theorem 3.6, (5) and (6), we know that

$$
F \cong \operatorname{Hom}_{S}\left(Q_{S},-\right) T^{\prime} \cong-\otimes_{S} P
$$

and

$$
G \cong \operatorname{Hom}_{T}\left(P_{T},-\right) S^{\prime} \cong-\otimes_{T} Q
$$

are naturally isomorphisms.

\section{Morita context for fair semigroups}

Definition 4.1. A right $S$-act $M$ is said to be strong $s$-unital, if for all $m_{1}, m_{2} \in M$, there exists $s \in S$ such that $m_{1} s=m_{2} s$.

Definition 4.2. A Morita context $\left(S, T,{ }_{S} P_{T},{ }_{T} Q_{S}, \tau, \mu\right)$ is unital, if ${ }_{S} P_{T}$ and ${ }_{T} Q_{S}$ are unitary biacts.

Ouyang etc. [15] obtained Morita-like equivalence based on Morita context for right-xst rings (Theorem 3.4). We also get similar result for right fair semigroups, but we need the assumption that $U(S)$ has common weak right local units. 
Theorem 4.3. Let $S$ and $T$ be two right fair semigroups such that $U(S)$ and $U(T)$ have common weak right local units. Assume $\left(S, T,{ }_{S} P_{T}, T_{T} Q_{S}, \tau, \mu\right)$ is a Morita context, where ${ }_{S} P_{T}$ and ${ }_{T} Q_{S}$ are strong s-unital and $\operatorname{Im} \tau=U\left(S_{S}\right)$ and $\operatorname{Im} \mu=U\left(T_{T}\right)$. We have the following conditions:

(1) $Q_{S}$ and $P_{T}$ are respectively generators of $U S$-Act and UT-Act.

(2) $P \otimes_{T} Q \stackrel{\tau}{\cong}{ }_{S} U\left(S_{S}\right)_{S}$ and $Q \otimes_{S} P \stackrel{\mu}{\cong}{ }_{T} U\left(T_{T}\right)_{T}$ as biacts.

Furthermore, if we define multiplications in $P \otimes_{T} Q$ and $Q \otimes_{S} P$ respectively by:

$$
(x \otimes y)\left(x^{\prime} \otimes y^{\prime}\right)=x \otimes \mu\left(y \otimes x^{\prime}\right) y^{\prime},(y \otimes x)\left(y^{\prime} \otimes x^{\prime}\right)=y \otimes \tau\left(x \otimes y^{\prime}\right) x^{\prime},
$$

where $x, x^{\prime} \in P$ and $y, y^{\prime} \in Q$, then $P \otimes_{T} Q$ and $Q \otimes_{S} P$ are semigroups, and $\tau$ and $\mu$ are semigroup isomorphisms.

(3) ${ }_{S} P_{T} \cong \operatorname{Hom}_{S}\left(Q_{S}, U\left(S_{S}\right)\right) U\left(T_{T}\right)$ and ${ }_{T} Q_{S} \cong \operatorname{Hom}_{T}\left(P_{T}, U\left(T_{T}\right)\right) U\left(S_{S}\right)$ as biacts.

(4) $U\left(T_{T}\right) \cong \operatorname{End}\left(Q_{S}\right) U\left(T_{T}\right)$ and $U\left(S_{S}\right) \cong \operatorname{End}\left(P_{T}\right) U\left(S_{S}\right)$ as semigroups.

(5) The functor pair $\left(-\otimes_{S} P,-\otimes_{T} Q\right)$ defines an equivalence $-\otimes_{S} P: U S$-Act $\rightleftharpoons$ UT-Act $:-\otimes_{T} Q$. That is, $S$ and $T$ are Morita-like equivalence semigroups.

(6) The functor pair $\left(\operatorname{Hom}_{S}\left(Q_{S},-\right) U\left(T_{T}\right), \operatorname{Hom}_{T}\left(P_{T},-\right) U\left(S_{S}\right)\right)$ defines an equivalence $H_{\text {Hom }}\left(Q_{S},-\right) U\left(T_{T}\right): U S-A c t \rightleftharpoons U T$-Act $: \operatorname{Hom}_{T}\left(P_{T},-\right) U\left(S_{S}\right)$. That is, $S$ and $T$ are Morita-like equivalence semigroups.

(7) The lattice of right ideals of $U\left(S_{S}\right)$ (resp., $U\left(T_{T}\right)$ ) is isomorphic to the lattice of subacts of $P_{T}\left(\right.$ resp., $\left.Q_{R}\right)$.

Furthermore, these induce lattice isomorphisms between the lattices of two-sided ideals of $U\left(S_{S}\right)$ (resp., $U\left(T_{T}\right)$ ) and the lattice of subacts of ${ }_{S} P_{T}\left(\right.$ resp., $\left.{ }_{T} Q_{S}\right)$.

Proof. For convenience, put $S^{\prime}=U\left(S_{S}\right)$ and $T^{\prime}=U\left(T_{T}\right)$.

(1) Define $f_{p}: Q \longrightarrow S^{\prime}$ by $q \mapsto \tau(p \otimes q)$. We have

$$
f_{p}(q s)=\tau(p \otimes q s)=\tau(p \otimes q) s .
$$

Hence, $f_{p} \in \operatorname{Hom}_{S}\left(Q, S^{\prime}\right)$. For all $s \in S^{\prime}=\operatorname{Im} \tau$, we have $s=\tau(p \otimes q)=f_{p}(q)$. Define $\theta: Q^{\operatorname{Hom}\left(Q, S^{\prime}\right)} \longrightarrow S^{\prime}$ by $q \longmapsto f_{p}(q)$. Therefore, $Q_{S}$ generates $S^{\prime}$.

Since $S^{\prime}$ is a generaor in $U S$-Act by Proposition 3.5, we have that $Q_{S}$ generates $M$ and $Q_{S}$ is a generator in $U S$-Act.

Similarly, $P_{T}$ is a generator in $U T$-Act.

(2) Suppose $x=q \otimes p, y=q^{\prime} \otimes p^{\prime}$ and $\mu(x)=\mu(y)$. As $P$ is strong $s$-unital, there exists $t \in T^{\prime}$ such that $p t=p^{\prime} t$. Set $t=\mu(v \otimes u)$. Then

$$
\begin{aligned}
x & =q \otimes p=q \otimes p t=q \otimes p \mu(v \otimes u)=q \otimes \tau(p \otimes v) u=q \tau(p \otimes v) \otimes u \\
& =\mu(q \otimes p) v \otimes u=\mu\left(q^{\prime} \otimes p^{\prime}\right) v \otimes u=q^{\prime} \otimes p^{\prime} \tau(v \otimes u)=y .
\end{aligned}
$$

So $\mu$ is injective. This implies that $\mu$ is a biact isomorphism and so $Q \otimes_{S} P \cong T^{\prime}$ as biact.

Next, to prove that $Q \otimes_{S} P$ is a semigroup, for any $x, x^{\prime}, x^{\prime \prime} \in P$ and $y, y^{\prime}, y^{\prime \prime} \in Q$, we have

$$
\begin{aligned}
\left((y \otimes x)\left(y^{\prime} \otimes x^{\prime}\right)\right)\left(y^{\prime \prime} \otimes x^{\prime \prime}\right) & =\left(y \otimes \tau\left(x \otimes y^{\prime}\right) x^{\prime}\right)\left(y^{\prime \prime} \otimes x^{\prime \prime}\right) \\
& =y \otimes \tau\left(x \otimes y^{\prime}\right) \tau\left(x^{\prime} \otimes y^{\prime \prime}\right) x^{\prime \prime} \\
& =(y \otimes x)\left(y^{\prime} \otimes \tau\left(x^{\prime} \otimes y^{\prime \prime}\right) x^{\prime \prime}\right) \\
& =(y \otimes x)\left(\left(y^{\prime} \otimes x^{\prime}\right)\left(y^{\prime \prime} \otimes x^{\prime \prime}\right)\right) .
\end{aligned}
$$

Hence, the multiplication satisfies the associative law. This proves that $Q \otimes_{S} P$ is a semigroup.

Similarly, $P \otimes_{T} Q$ is also a semigroup.

Clearly, $\mu$ and $\tau$ preserve the multiplication operations, respectively. So $\mu$ and $\tau$ are semigroup isomorphisms. 
(3) Define $\theta: P \rightarrow \operatorname{Hom}_{S}\left(Q_{S}, S_{S}^{\prime}\right)$ by $p \mapsto \theta(p)$ for $p \in P$, where $\theta(p)(q)=\tau(p \otimes q)$ for $q \in Q$.

Clearly, $\theta$ is a morphism.

Suppose $\theta(p)=\theta\left(p^{\prime}\right)$, we have $\tau(p \otimes q)=\tau\left(p^{\prime} \otimes q^{\prime}\right)$, for all $q \in Q$. Since $P$ is strong $s$-unital, there exists $t \in T^{\prime}$ such that $p t=p^{\prime} t$, where $t=\mu(v \otimes u)$. Hence

$$
p=p t=p \mu(v \otimes u)=\tau(p \otimes v) u=\tau\left(p^{\prime} \otimes v\right) u=p^{\prime} \mu(v \otimes u)=p^{\prime} t=p^{\prime} .
$$

It follows that $\theta$ is injective. Thus $\operatorname{Im} \theta \cong P$ is a unitary $S$-T-subbiact of $\operatorname{Hom}_{S}\left(Q_{S}, S_{S}^{\prime}\right)$. From Proposition 3.1, we know that $\operatorname{Hom}_{S}\left(Q_{S}, S_{S}^{\prime}\right) T^{\prime}$ is the largest unitary right $T$-subact of $\operatorname{Hom}_{S}\left(Q_{S}, S_{S}^{\prime}\right)$. Then $\operatorname{Im} \theta \subseteq \operatorname{Hom}_{S}\left(Q_{S}, S_{S}^{\prime}\right) T^{\prime}$. For $f \in \operatorname{Hom}_{S}\left(Q_{S}, S_{S}^{\prime}\right) T^{\prime}$, since $T^{\prime}$ is $s$-unital, there exists $e \in T^{\prime}$ such that $f e=f$. Suppose $e=\mu(y \otimes x)$, where $y \in Q$ and $x \in P$. Let $x^{\prime}=f(y) x$. Then $x^{\prime} \in P$ and

$$
\begin{aligned}
\theta\left(x^{\prime}\right)(y) & =\theta(f(y) x)(y)=\tau(f(y) x \otimes y) \\
& =f(y) \tau(x \otimes y)=f(y \tau(x \otimes y)) \\
& =f(\mu(y \otimes x) y)=f(e y) \\
& =(f e)(y)=f(y) .
\end{aligned}
$$

Therefore, $f=\theta\left(x^{\prime}\right)$ and $\operatorname{Im} \theta=\operatorname{Hom}_{S}\left(Q_{S}, S_{S}^{\prime}\right) T^{\prime}$. Hence, ${ }_{S} P_{T} \cong \operatorname{Hom}_{S}\left(Q_{S}, S_{S}^{\prime}\right) T^{\prime}$.

Similarly, we can prove ${ }_{T} Q_{S} \cong \operatorname{Hom}_{T}\left(P_{T}, T_{T}^{\prime}\right) S^{\prime}$.

(4) Define $\lambda: T^{\prime} \rightarrow \operatorname{End}\left(Q_{S}\right)$ by $\lambda(t)(y)=t y$ for $y \in Q$. For $t, u \in T^{\prime}$, suppose $\lambda(t)=\lambda(u)$. Then $t y=\lambda(t)(y)=\lambda(u)(y)=u y$ for $y \in Q$. By assumption, $\exists e \in T^{\prime}$ such that $t e=t$ and $u e=u$. Let $e=\mu(y \otimes x)$, where $y \in Q, x \in P$. Then

$$
t=t e=t \mu(y \otimes x)=\mu(t y \otimes x)=\mu(u y \otimes x)=u \mu(y \otimes x)=u e=u .
$$

This proves that $\lambda$ is injective and $\operatorname{Im} \lambda \cong T^{\prime}$. Hence, $\operatorname{Im} \lambda$ is a subsemigroup of $\operatorname{End}\left(Q_{S}\right)$. Since $\operatorname{Im} \lambda$ is a right unitary $T^{\prime}$-act, $\operatorname{Im} \lambda$ is a subact of $\operatorname{End}\left(Q_{S}\right) T^{\prime}$ by Proposition 3.2. For all $f \in \operatorname{End}\left(Q_{S}\right) T^{\prime}$, there exists $e \in T^{\prime}$ such that $f e=f$. Suppose $e=\mu(y \otimes x)$, where $y \in Q$ and $x \in P$. Set $t^{\prime}=\mu(f(y) \otimes x)$. For all $q \in Q$, we have

$$
\begin{aligned}
\lambda\left(t^{\prime}\right)(q) & =t^{\prime} q=\mu(f(y) \otimes x) q=f(y) \tau(x \otimes q) \\
& =f(y \tau(x \otimes q))=f(\mu(y \otimes x) q)=f(e q)=(f e)(q)=f(q) .
\end{aligned}
$$

Hence, $f=\lambda\left(t^{\prime}\right)$ and $\operatorname{Im} \lambda=\operatorname{End}\left(Q_{S}\right) T^{\prime}$. Then $T^{\prime} \cong \operatorname{End}\left(Q_{S}\right) T^{\prime}$ as semigroup.

Similarly, $U\left(S_{S}\right) \cong \operatorname{End}\left(P_{T}\right) U\left(S_{S}\right)$ as semigroup.

(5) Firstly, we prove $-\otimes_{S} P \otimes_{T} Q \cong I_{U S \text {-Act }}$. Since $P \otimes Q \cong S^{\prime}$, we can prove

$$
M \otimes_{S} S^{\prime} \cong M, \text { for all } M_{S} \in U S \text {-Act. }
$$

Define $\sigma: M \otimes_{S} S^{\prime} \rightarrow M$ by

$$
\sigma(m \otimes s)=m s, \text { for } m \otimes s \in M \otimes_{S} S^{\prime} .
$$

Clealy, $\sigma$ is a morphism. By Proposition 3.5, $M$ is a right unitary $S$-act. Hence, $\sigma$ is surjective.

Assume $\sigma(m \otimes s)=m s=m^{\prime} s^{\prime}=\sigma\left(m^{\prime} \otimes s^{\prime}\right)$. Since $S^{\prime}$ has common weak right local units, there exists $e \in S^{\prime}$ such that $s e=s^{\prime} e$. Hence,

$$
m \otimes s=m \otimes s e=m s \otimes e=m^{\prime} s^{\prime} \otimes e=m^{\prime} \otimes s^{\prime} e=m^{\prime} \otimes s^{\prime} .
$$

So $\sigma$ is injective. Therefore,

$$
\left(M \otimes_{S} P\right) \otimes_{T} Q \cong M \otimes_{S}\left(P \otimes_{T} Q\right) \stackrel{I \otimes \tau}{\cong} M \otimes_{S} S^{\prime} \stackrel{\sigma}{\cong} M .
$$


The naturality is obvious. So we get the desired result.

Similarly, we can get $-\otimes_{T} Q \otimes_{S} P \cong I_{U T \text {-Act. }}$.

(6) By (5) and Theorem 3.6, we can prove (6).

(7) Set

$$
\begin{gathered}
\mathcal{J}=\left\{I \mid I \text { is a right ideal of } S^{\prime}\right\} ; \\
\mathcal{N}=\{N \mid N \text { is a subact of } P\} .
\end{gathered}
$$

Define $f: \mathcal{J} \longrightarrow \mathcal{N}$ by $I \longmapsto I P$, where $I P=\{i p \mid i \in I, p \in P\}$ and $g: \mathcal{N} \longrightarrow \mathcal{J}$ by $N \longmapsto \tau\left(N \otimes_{T} Q\right)$, where $\tau\left(N \otimes_{T} Q\right)=\{\tau(n \otimes q) \mid n \in N, q \in Q\}$.

Clearly, $f$ and $g$ are inverse lattice isomorphisms between $\mathcal{J}$ and $\mathcal{N}$.

Similarly, the lattice of right ideals of $T^{\prime}$ is isomorphic to the lattice of subacts of $Q_{S}$.

Obviously, these isomorphisms induce lattice isomorphisms between the lattice of ideals of $S^{\prime}$ (resp., $\left.T^{\prime}\right)$ and the lattice of subacts of ${ }_{S} P_{T}\left(\right.$ resp., $\left.{ }_{T} Q_{S}\right)$.

Acknowledgment. The author is grateful to the referees for their careful reviewing and valuable comments which improve the paper highly.

\section{References}

[1] G. Abrams, Morita equivalence for rings with local units, Commun. Algebra, 11, 801-837, 1983.

[2] P.N. Ánh and L.Márki, Morita equivalence for rings without identity, Tsukuba J. Math. 11, 1-16, 1987.

[3] B. Banaschewski, Functors into categories of M-sets, Abh. Math. Sem. Univ. Hamburg, 38, 49-64, 1972.

[4] K.R. Fuller, Density and equivalence, J. Algebra 29, 528-550, 1974.

[5] J.L. García and L.Marín, Rings having a Morita-like equivalence, Commun. Algebra, 27, 665-680, 1999.

[6] J.M. Howie, Fundamentals of semigroup theory, Clarendon press, Oxford, 1995.

[7] M. Kilp, U. Knauer and A.V. Mikhalev, Monoids Acts and Categories, Degruyter Expositions in Mathematics, 2000.

[8] U. Knauer, Projectivity of acts and Morita equivalence of monoids, Semigroup Forum 3, 359-370, 1972.

[9] V. Laan and L. Márki, Strong Morita equivalence of semigroups with local units, J. Pure Appl. Algebra, 215, 2538-2546, 2011.

[10] V. Laan and L. Márki, Morita invariants for semigroups with local units, Monatsh. Math. 166, 441-451, 2012.

[11] V. Laan and L. Márki, Fair semigroups and Morita equivalence, Semigroup Forum, 92, 633-644, 2016.

[12] M.V. Lawson, Morita equivalence of semigroups with local units, J. Pure Appl. Algebra 215, 455-470, 2011.

[13] H. Liu, Morita equivalence based on Morita context for arbitrary semigroups, Hacet. J. Math. Stat. 45 (4), 1083-1090, 2016.

[14] B.Y. Ouyang and W.T. Tong, Morita context and Morita-like equivalence for the xst-rings, Acta Math. Sinica (English Series), 19, 371-380, 2003.

[15] B.Y. Ouyang, L.R. Zhou and W.T. Tong, Characterizations of Morita-like Equivalences for right xst-rings, Algebra Colloquium, 14, 85-95, 2007.

[16] S. Talwar, Morita equivalence for semigroups, J. Aust. Math. Soc. (Series A), 59, 81-111, 1995.

[17] Y.H. Xu, K.P. Shum and R.F. Turner-Smith, Morita-like equivalence of infinite matrix subrings, J. Algebra, 159, 423-435, 1993. 